cies - these are the "natural frequencies" of the system and show up as peaks in the spectral density. According to the theory, the key signature is how this peak depends on the input noise strength. If the peak grows rapidly with increasing noise, then stochastic resonance should occur.

Now, the natural next step is to complete the picture by drawing the connection between these considerations and the "old" stochastic resonance associated with the bistable systems everyone else had studied. This could be done, but the Lancaster group noticed something interesting: there was nothing in the analysis that required bistability. They reasoned that bistability wasn't absolutely necessary, and that stochastic resonance could in principle occur in a monostable system. And furthermore, they already knew of "a promising monostable candidate" which fulfilled the necessary requirements according to linear response theory. This was a well worn workhorse known as the damped Duffing equation, which describes the motion of a mass tied to an imperfect (nonlinear) spring and subject to viscous damping. For this problem, the oscillator is also subject to a combination of periodic and random forces.

Rather than construct a mechanical realisation, the Lancaster group built an electronic circuit which obeys the same equation of motion. On the one hand, it was a straightforward matter to measure the spectral density function; however, for this equation they were also able to calculate the function analytically, and so test the predictions of their theory in detail. The experiments showed that hallmark signature of stochastic resonance: as the noise intensity was gradually increased from a low level, the response at the signal frequency first improved, then passed through a maximum before falling off at large values of the noise input. The data were found to be in close agreement with the theoretical predictions.

Finally, the Lancaster group addresses the name stochastic resonance itself. Is their new example essentially distinct, or is it more naturally viewed as a close relative of earlier examples of stochastic resonance? Though such discussions are peculiar to see in the technical literature, the issue of appropriate terminology has been a sticking point in this field. The problem is whether this is truly a resonance phenomenon as taught in introductory physics classes, in which forcing at just the right frequency results in a large energy transfer, as depicted in the famous film footage of the Tacoma Narrows Bridge collapse. In previous studies the connection was accepted as relatively weak, but the name was already established, so there it was. But in an interesting twist, the Lancaster group points out that their "new" example is truly related to classic resonance. Nevertheless, they decide that the similarities with the "old" examples outweigh the differences, since the key fundamental property is retained: the addition of random noise can improve the sensitivity to a periodic stimulus.

\title{
Order through disorder: entropy strikes back
}

From Daan Frenkel in the FOM Institute for Atomic and Molecular Physics, Amsterdam, The Netherlands

THE second law of thermodynamics states that the entropy of an isolated system increases until it reaches a maximum. Entropy is traditionally associated with disorder but recent experiments, computer simulations and theories have illustrated how the disordering effect of entropy can lead to highly ordered superstructures in certain alloys.

Statistical mechanics can be used to give a "microscopic" interpretation of entropy and the second law: in suitable units, entropy is simply equal to the logarithm of the number of (quantum) states accessible to the system. If we constrain a system to be in a single state, its entropy will be zero. If we release this constraint and allow the system to explore all other states with the same energy, the entropy will increase until the system is equally likely to be in any one of these states. At that point the entropy is at a maximum and the system has reached equilibrium. Of course, in the process, we have lost information about the system: initially, we knew exactly what state it was in, but after equilibration, the system can be in any one of a large number of states. We have gone from an ordered initial situation to a more disordered final situation. In this sense, entropy is a measure for the amount of disorder in a system.

Can we "see" order or disorder? Strictly speaking, the answer is no, because we cannot see the number of accessible states of a system. However, we have another, more intuitive notion of order and disorder based on things that we can see. We call a crystal ordered because its constituent particles are constrained to be near specific points in space (lattice sites). A fluid, where no such constraints apply, is called disordered. Our intuitive notion about order and disorder suggests that a system with a given density and energy should have a higher entropy in the fluid phase than in the crystalline phase, and that freezing would result in a decrease of entropy. The second law of thermodynamics tells us that this can only happen if this decrease in entropy is offset by a larger increase in entropy of some other part of the Universe. This can happen if the energy of the crystalline phase is lower than that of the liquid phase such that the system can release heat on freezing, thereby increasing the entropy of the rest of the Universe. This simple-minded description of the thermodynamics of freezing explains why, for a long time, it was commonly thought that attractive forces between molecules are essential for crystallisation: a crystal can form because the lowering of the potential energy of the system upon solidification "pays for" the decrease in entropy.

It was therefore a great surprise when, in the $1950 \mathrm{~s}$, computer simulations indicated that a fluid of hard spheres could freeze. Hard spheres do not have any interactions so the potential energy of such a system is always zero. Hence, if crystallisation occurs, this can only mean that the entropy of the crystalline solid is higher than that of the fluid! Although, at present, the hard-sphere freezing transition is well established and non-controversial, this was certainly not the case when it was first reported. During a round-table discussion on the topic in 1957 George Uhlenbeck, the chairman, asked the experts on the panel (including two other Nobel laureates) to vote on the existence of the hard-sphere freezing transition. The vote was even and Uhlenbeck closed the discussion by saying: "I am quite sure that the transition goes a little bit against intuition: that is why so many people have difficulty with it, and surely I am one of those. But this transition - it still might be true, you know - and I don't think one can decide by general arguments."

Yet, in retrospect, we can understand the hard-sphere freezing as follows: a naive picture of a solid is a model in which all the molecules are confined to cells centred on lattice sites. Confining the molecules to cells decreases entropy (we call this contribution "configurational"). However, we also gain entropy because a molecule has more free volume to move in this cell than it had in the fluid (the molecules are more congested in a dense fluid than in a solid of the same density). At sufficiently high densities, the gain in entropy due to this increase in free volume exceeds the loss in configurational entropy, and entropy therefore favours crystallisation. In other words, an increase in macroscopic order is driven by an increase of microscopic disorder. In fact, numerous ordering transitions are now known to be driven by entropy alone.

The complexity of the order that can be induced by entropy only became clear recently when Paul Bartlett and $\mathrm{R} \mathrm{H}$ Ottewill from Bristol University and Peter Pusey from Edinburgh University reported experiments on the crystallisation of a colloidal suspension of a mixture of "large" and "small" polymethylmethacrylate (PMMA) particles with a size ratio of 
0.58 (Phys. Rev. Lett. (1992) 68 3801). These mesoscopic particles have a steep repulsive interaction, not unlike hard spheres (which are not permitted to overlap). Bartlett and co-workers observed that this mixed colloid would form a crystal with a so-called $\mathrm{AB}_{13}$ structure. This structure is familiar in metal alloys (e.g. $\mathrm{NaZn}_{13}$ ) and consists of a simple cubic lattice of $\mathrm{A}$ particles. In the middle of each A-cube is a B particle surrounded by an icosahedral cluster (a figure with 20 faces and 12 vertices) of other Bs. The full unit cell consists of eight such sub-cells with neighbouring icosahedra alternating in orientation by 90 .

These findings immediately raised two questions: 1) is the observed $\mathrm{AB}_{13}$ phase stabilised by entropy alone, or is the fact that PMMA particles are not hard spheres crucial? and 2) can we understand why this phase should form? A definitive answer to the first question can only come from computer simulations and recent results from an extensive numerical study by Matthew Eldridge and co-workers of Oxford University indicate that entropy alone can indeed account for the stability of the $\mathrm{AB}_{13}$ structure (Mol. Phys. in press). In another recent paper Hong $\mathrm{Xu}$ of the Centre Européen de Calcul Atomique et Moléculaire at Orsay and Marc Baus of the Free University of Brussels consider both questions from a different point of view $(\mathcal{F}$. Phys.: Condensed Matter (1992) 4 L663).

$\mathrm{Xu}$ and Baus use classical densityfunctional theory, i.e. the best analytic theory of freezing to date, to estimate the stability of the $\mathrm{AB}_{13}$ phase in a mixture of large and small hard spheres. Their calculations confirm that, for a size ratio of 0.58 , there is a density range where the $\mathrm{AB}_{13}$ solid is more stable than the fluid mixture, the pure $\mathrm{A}$ and $\mathrm{B}$ solids, and the $\mathrm{AB}_{2}$ compound.

So why is $\mathrm{AB}_{13}$ the stable solid structure? Surprisingly it is not the free volume that is responsible - both the pure $\mathrm{A}$ and $\mathrm{B}$ phases and the $\mathrm{AB}_{2}$ structure can fill space more efficiently, and hence the free volume would favour those phases over $\mathrm{AB}_{13}$. The $\mathrm{AB}_{13}$ phase is stable because it has a higher configurational entropy. And in a mixture, this configurational entropy is closely related to the entropy of mixing. In the words of $\mathrm{Xu}$ and Baus: "the larger entropy of mixing of the $\mathrm{AB}_{13}$ structure relative to that of the competing structures is responsible for its stability." I guess that George Uhlenbeck would have found this more than "a little counter-intuitive".

\section{Use the reader enquiry card for fast, free information on advertised products}

\section{Fibre sensors promise improved safety underground}

From Bob Lieberman at Physical Optics Corporation, Torrance, California, and AT\&T Bell Laboratories, Murray Hill, New Jersey, US

A CONTINUING hazard in coal mines is the build up of methane gas, which can form an explosive mixture in air with disastrous results. Although ignition sources in mines are limited, explosions and uncontrolled methane fires still occur far too often. Because methane is odourless and colourless, and because the lower explosive limit of methane in air is so low ( $5 \%$ concentration by weight) that miners feel no "shortness of breath" when concentrations reach dangerous levels, it is impossible to tell when methane is present without using some sort of detection apparatus.

Now, a new class of methane detection system has emerged, incorporating optical fibres to offer improved reliability and, potentially, lower cost than either of the existing techniques. Canaries aside, the only other detection methods are based on the catalysis of methane at the surface of a specialised electrode, or on spectroscopic detection using infrared optical absorption. Unfortunately, the electronic systems are unreliable, and the available optical systems are often expensive and cumbersome, since they rely on Fourier transform infrared (FTIR) spectrometers and long-path spectroscopic cells with precisely aligned reflective elements.

The new approach uses optical fibres to carry light between the spectrometer and the test region. A group of researchers from British Gas, AEA Harwell, British Telecom, Sieger, and headed by Strathclyde University, UK, has now made a significant advance that could realise practical fibrebased methane monitoring systems capable of in situ measurements in previously inaccessible locations (B Culshaw et al. Electron. Lett. (1992) 28 2232).

Fibres allow the optical source, and all electronic components, to be located hundreds of metres from the monitoring area. Thus, there is no chance of heat or electrical discharges from the monitoring system accidentally triggering an explosion of hazardous mixtures. An added benefit is that, using a simple optical switch, it is possible to address many monitoring locations with a single spectrometric readout system. To date, fibre-based monitoring equipment generally consisted of a remotely located spectroscopic cell connected to a fibre, and optical measurements either proceeded by FTIR, or by means of more innovative, and potentially less expensive, techniques such as modulation spectroscopy (J P Dakin et al. f. Opt. Sens. (1987) 2 261).

The Strathclyde collaboration, which began in 1988, has concentrated on replacing the spectroscopic cell altogether with an optical fibre. The key to accomplishing this is a specially designed "D-fibre" (see figure 1a), which allows light carried by the fibre core to interact with its surroundings. This approach has the benefit of requiring virtually no optical alignment in the sensing region, and actually measures methane concentrations over the entire length of the fibre.

Although, to a first approximation, optical energy carried by a fibre is confined to the core by total internal reflection, an "evanescent" electromagnetic field associated with the core-guided light actually extends into the fibre cladding. The amount of energy carried by this field depends on the relative indices of refraction of the core and cladding, and on the

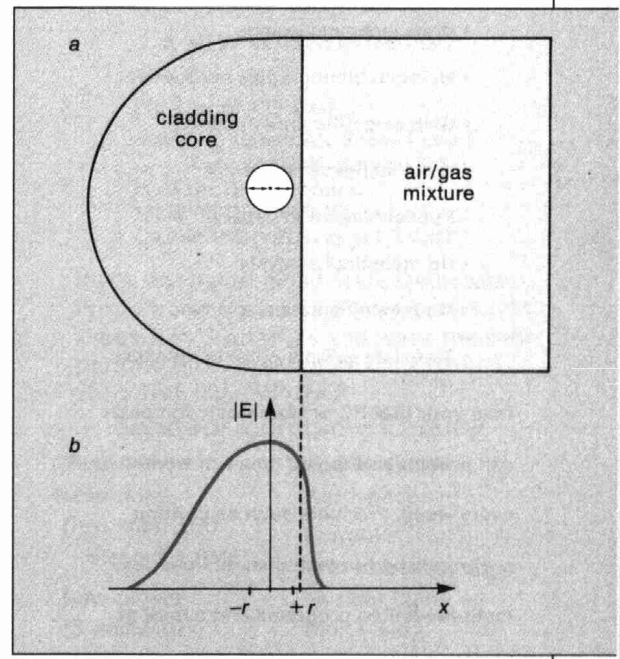

1 (a) D-fibre with core radius $r$; (b) plot of electromagnetic field intensity versus distance from centre of core shows how a substantial amount of optical energy extends beyond the surface of the fibre, interacting with the air/gas mixture

core diameter. When the core of the fibre is small, as in the case of the "single-mode" fibres used at Strathclyde, the evanescent field is large.

Telecommunications researchers discovered nearly 20 years ago that the absorption of light from the evanescent field by cladding impurities would cause significant transmission losses. Since then they have worked to minimise this effect. Now, in an interesting turnabout, the Strathclyde collaboration has fabricated fibres that actually maximise the interaction of the evanescent field with material outside the core. In fact, the new D-fibre has the core so near its surface that the field extends beyond the boundaries of the fibre (figure $1 b)$; as a result, the optical properties of 